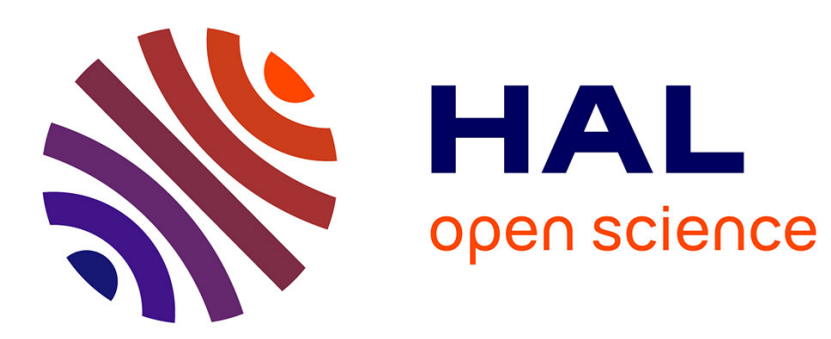

\title{
Essai de phylogénie d'espèces proches : cheval, âne et mulet par les techniques modernes de marquage

\author{
Colette Laurent, M. Cl. Biemont
}

\section{To cite this version:}

Colette Laurent, M. Cl. Biemont. Essai de phylogénie d'espèces proches: cheval, âne et mulet par les techniques modernes de marquage. Annales de génétique et de sélection animale, 1977, 9 (4), pp.535-536. hal-00892926

\section{HAL Id: hal-00892926 https://hal.science/hal-00892926}

Submitted on 1 Jan 1977

HAL is a multi-disciplinary open access archive for the deposit and dissemination of scientific research documents, whether they are published or not. The documents may come from teaching and research institutions in France or abroad, or from public or private research centers.
L'archive ouverte pluridisciplinaire HAL, est destinée au dépôt et à la diffusion de documents scientifiques de niveau recherche, publiés ou non, émanant des établissements d'enseignement et de recherche français ou étrangers, des laboratoires publics ou privés. 


\title{
The translocation $1 / 29$ \\ in Swedish Red-White cows imported from Sweden to Hungary
}

\author{
I. GUSTAVSSON and A. KOVACS * \\ Royal Vet. Coll., Stockholm, Sweden \\ * Central Station for Artificial Insemination, \\ Budapest, Hungary
}

300 Swedish Red-White heifers have been imported without having asked for any guarantee of their normal karyotypes. I6 of them - having a I/29 carrier maternal grandfather - were evaluated. 3 cows and one calf born in Hungary were found as heterozygous for the $1 / 29$ centric fusion.

\section{Planches descriptives des chromosomes du cheval (Equus caballus) et de l'âne (Asinus asinus) (Analyse en bandes $\mathrm{R}$ )}

\section{Cl. BIEMONT et Colette LAURENT}

Laboratoire de Cytogénétique, Institut Pasteur de Lyon, 77, rue Pasteur, 69365 Lyon Cedex 2 (France)

L'analyse de métaphases et de prométaphases observées après techniques de dénaturation thermique ménagée, nous a permis de déterminer préciséntent la disposition des bandes $\mathrm{R}$ de chaque chromosome de ces deux espèces.

Il nous a paru utile d'établir un prototype de planches descriptives de ces bandes $R$ pour ces deux espèces domestiques; ces planches pouvant servir de référence pour le diagnostic d'anomalies chromosomiques chez ces animaux.

\section{Les bandes $\mathbf{R}$ des chromosomes des espèces bovine, ovine et caprine}

\author{
M. FRANCK, J. FROGET, et M. C. NAIN \\ École Nationale Vétérinaive de Lyon, 2, quai Chaveau, 69 Lyon (France)
}

L'étude des chromosomes par la méthode des bandes $\mathrm{R}$ donne des résultats in téressants, à condition de modifier légèrement la méthode de culture utilisée pour l'espèce humaine, de même que la technique de dénaturation.

Les auteurs proposent une séquence des bandes $R$ de chaque paire de chromosomes, pour les espèces bovine, ovine et caprine, qui puisse servir ultérieurement de référence.

\section{Essai de phylogénie d'espèces proches : cheval, âne et mulet par les techniques modernes de marquage}

\author{
Colette LAURENT et M. Cl. BIEMONT
}

Laboratoire de cytogénétique, Institut Pasteur de Lyon, 77, rue Pasteur, 69365 Lyon Cedex 2, France

Depuis longtemps déjà, on sait que les remaniements chromosomiques jouent un rôle important dans l'évolution et la spéciation.

Nous avons étudié les équidés et plus particulièrement le cheval (Equus caballus) et l'âne ( $A$ sinus asinus) dont les hybrides sont viables, les nombres chromosomiques voisins, ce qui laissait supposer à priori que leurs caryotypes seraient peu différents.

Entre les 3 I paires d'autosomes du cheval et les 30 paires de l'âne, il ne semble exister que peu de correspondance. Ces différences chromosomiques importantes sont sans doute la cause de la stérilité de l'hybride. 
La comparaison des chromosomes $X$ permet de supposer qu'ils ont une origine commune et deux remaniements chromosomiques permettent de passer de l'un à l'autre.

De nombreuses formes intermédiaires ont dû exister entre les deux espèces actuelles, mais les possibilités d'hybridation montrent que les remaniements chromosomiques ont eu un rôle cloisonnant relativement modéré.

\title{
Essai de phylogénie chez quelques actiodactyles à partir des bandes $R$
}

\author{
M. THERET, J. FROGET, M. FRANCK et M. C. NAIN
}

\author{
École Nationale Vétérinaire de Lyon, 2, quai Chaveau, 69ooo Lyon, France
}

Les chromosomes de bovins et de caprins, dénaturés par la méthode des bandes $R$, présentent une grande similitude, au plan de leur nombre et de leur structure; il s'agit de 28 paires d'autosomes télocentriques. La différence la plus marquante entre les deux caryotypes concerne le chromosome $\mathbf{X}$ des caprins, qui est télocentrique, alors que celui des bovins est submétacentrique. En outre, les chromosomes : II, I $4,18,25,26,28$, et l'Y, sont très légèrement différents d'une espèce à l'autre.

Les chromosomes de l'espèce ovine sont au nombre de 54. Trois paires d'autosomes submétacentriques résulteraient de la fusion centrique entre les chromosomes I et 4,2 et 8,5 et 9 . Le caryotype de l'espèce ovine, pour les autres chromosomes, est très proche de celui de l'espèce caprine.

Ovis aries, ovis musimon et leur hybride, ne présentent pas de différences marquantes quant à leur caryotype.

\section{Chromosomal studies on Cultured Fetal Fluid Cells collected by Transsacrostiatic Amniocentesis from Heifers during the Third Month of Pregnancy}

\author{
E. I. SINGH, M. D. EAGLESOME, W. C. D. HARE and D. MITCHELL (*)
}

\author{
Animal Pathology Division, Health of Animals Branch, \\ Agricultuve Canada, Animal Diseases Research Institute (E), \\ Box II 300 , Station $H$, Ottawa, Ontario, Canada, $\mathrm{Kz} \mathrm{H} 8 \mathrm{P9}$ \\ $\left(^{*}\right)$ Animal Diseases Research Institute (W) P.O. Box 640, Lethbridge, Alberta, Canada TIJ $3 Z_{4}$
}

Attempts were made to collect fetal fluids from 37 pregnancies in 34 heifers during the third month of pregnancy by inserting an aspirating needle through the sacrosciatic ligament and uterine wall into the fetal sac.

In ro trial attempts, six samples were collected and the cells set up in culture; four cases of fetal death occurred. Subsequently, in 27 test attempts, cells from 23 of 25 samples collected were set up in culture; three cases of fetal death occurred. Eleven cell cultures were also set up from fluids obtained from slaughterhouse material.

Sufficient growth for chromosomal analysis was obtained from 28 of 40 cultures. Anatomical confirmation of sex was made in 23 of these. Confirmation was not possible in three cases due to absorption or maceration of the fetus, but the chromosomal sex of one of these was male and, therefore, had to be correct. The sex of the other two cases was predicted to be female and found to be male.

Contamination of fetal fluid samples by maternal cells was a constant possibility which could lead to the misinterpretation of results. To minimize this, the first $\mathrm{I}-2 \mathrm{ml}$ of fetal fluids aspirated were discarded, multiple cultures were set up from each sample and, when male cells were not observed, numerous metaphase spreads from several passages of each culture were studied.

Twelve cultures failed due to incubator failure, bacterial or fungal contamination, or too few culturable cells. 